\title{
Magnetic Properties of Synthetic Gehlenite Glass Microspheres
}

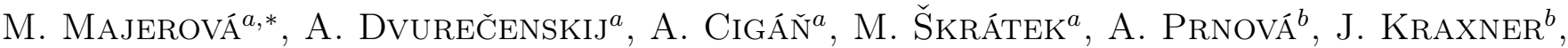 \\ D. GALUSEK ${ }^{b}$ AND J. MAŇKA ${ }^{a}$ \\ ${ }^{a}$ Department of Magnetometry, Institute of Measurement Science, SAS, \\ Dúbravská cesta 9, 84104 Bratislava, Slovakia \\ ${ }^{b}$ Vitrum Laugaricio - Joint Glass Center of the IIC SAS, TnU AD, FCHTP STU and Rona, a.s., \\ Študenstská 2, 91150 Trenčín, Slovakia
}

\begin{abstract}
In the paper, gehlenite amorphous microspheres were prepared by the flame synthesis of a powder precursor. In the first step, the precursor was prepared from a stoichiometric mixture of $\mathrm{CaCO}_{3}, \mathrm{Al}_{2} \mathrm{O}_{3}$ and $\mathrm{SiO}_{2}$ by a standard solid-state reaction method. Next, the precursor was sprayed into a $\mathrm{CH}_{4}-\mathrm{O}_{2}$ flame with the temperature of around $2200{ }^{\circ} \mathrm{C}$ and molten droplets of synthetic gehlenite were rapidly cooled by distilled water. Structural and detailed magnetic properties were studied by the optical microscopy, X-ray diffraction and QD SQUID magnetometer. The gehlenite microspheres show a complex magnetic behaviour that is a function of the temperature and the magnetic field, e.g. diamagnetism and paramagnetism at $300 \mathrm{~K}$ and $2 \mathrm{~K}$, respectively.
\end{abstract}

DOI: 10.12693/APhysPolA.131.699

PACS/topics: 81.05.Kf, 75.50.Kj, 91.60.Pn, 75.20.--g, 75.50.Lk

\section{Introduction}

Gehlenite, the calcium aluminosilicate $\left(\mathrm{Ca}_{2} \mathrm{Al}_{2} \mathrm{SiO}_{7}\right)$, is a refractory rock-forming mineral. The gehlenite phase regularly occurs in the ashes from lignite, biomass fired power plants, in red mud and fly ash in the aluminium industries. It can be found in various ceramic materials, as building materials, cookware or as implemented in cement composites. Gehlenite is used in the preparation of biosoluble glass fibres and ceramics. It is a suitable host for optically active dopants, with potential applications as phosphors for LEDs.

In general, glasses can be characterized as a material that lacks long range ordering atoms. It is known that calcium aluminosilicate glasses are composite from building blocks of tetrahedral $\mathrm{SiO}_{4}$ and $\mathrm{AlO}_{4}$ units forming of glass network. $\mathrm{Ca}^{2+}$ cation compensates the excess of negative charge of the tetrahedral $\left(\mathrm{AlO}_{4}\right)^{1-}$ units, considering oxidation state of cation in the units of $\mathrm{Al}^{3+}[1]$.

In the paper, we prepared synthetic amorphous gehlenite microspheres and studied their structural, optical and magnetic properties.

\section{Experimental}

In the first step, the powder precursor was prepared from a stoichiometric mixture of high-purity powders of $\mathrm{CaCO}_{3}, \mathrm{Al}_{2} \mathrm{O}_{3}$, and $\mathrm{SiO}_{2}$. At first, the starting mixture was homogenised in an agate mill and calcinated in a two-step process at $650^{\circ} \mathrm{C}$ for $4 \mathrm{~h}$ following annealing at $1000^{\circ} \mathrm{C}$ for $4 \mathrm{~h}$. The pre-reacted precursor was milled

*corresponding author; e-mail: melinda.majerova@savba.sk and sieved trough a $40 \mu \mathrm{m}$ polyethylene sieve. Glass microspheres were prepared by dosing the powder precursor into $\mathrm{CH}_{4}-\mathrm{O}_{2}$ flame with temperature of about $2200^{\circ} \mathrm{C}$, wherein the powder particles were melted. To achieve a higher cooling rate of the molten droplets, they were showered by gently nebulized distilled water. After separating, the microspheres were annealed in an oven at $650{ }^{\circ} \mathrm{C}$ for $1 \mathrm{~h}$ in order to remove any possible organic impurities generated by the incomplete combustion of methane. Powder X-ray diffraction was performed by means of a PANalytical Empyrean diffractometer configured with a $\mathrm{Cu} K_{\alpha}$ radiation.

The morphology of microspheres was obtained by $\mathrm{Ni}$ kon Eclipse ME 600 transmitted light microscopy.

The mass magnetization $M^{\prime}$ (corrected to sample holder) at $300 \mathrm{~K}$ and $2 \mathrm{~K}$ and temperature dependences of the ZFC/FC dc molar magnetic susceptibility were measured by the QD SQUID magnetometer MPMS XL-7. The molar (per formula unit) susceptibilities $\chi$ were corrected to the effect of the sample holder and to the diamagnetism of inner electrons using the tabulated values of diamagnetic susceptibilities of cations and anions given by Bain and Berry [2], considering $\mathrm{Ca}^{2+}, \mathrm{Al}^{3+}, \mathrm{Si}^{4+}$ and $\mathrm{O}^{2-}$ ions, in the first approximation.

\section{Results}

The optical photograph of gehlenite microspheres is shown in Fig. 1. The photograph shows the spherical transparent particles with a relatively high diameter size distribution. However, partially melted or remelted particles can also be identified in the sample.

The results of the X-ray powder diffraction measurements (corrected for background) both with XRD patterns of crystalline gehlenite sample obtained with Crystallography Open Database are presented in Fig. 2. The 


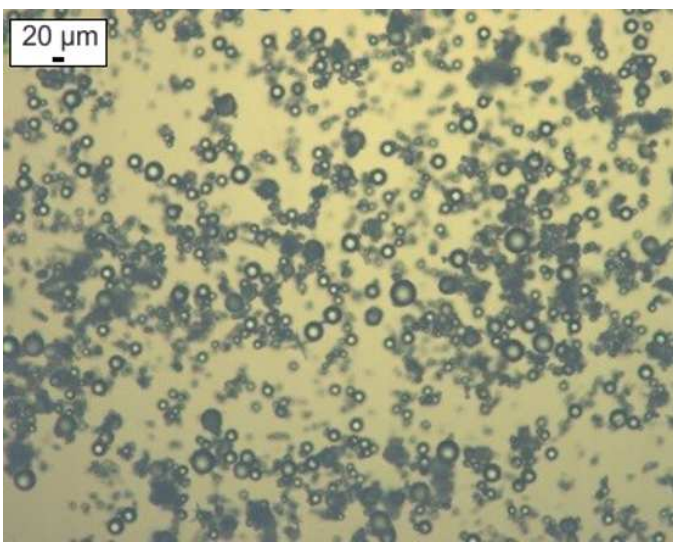

Fig. 1. Optical photograph of gehlenite microspheres.

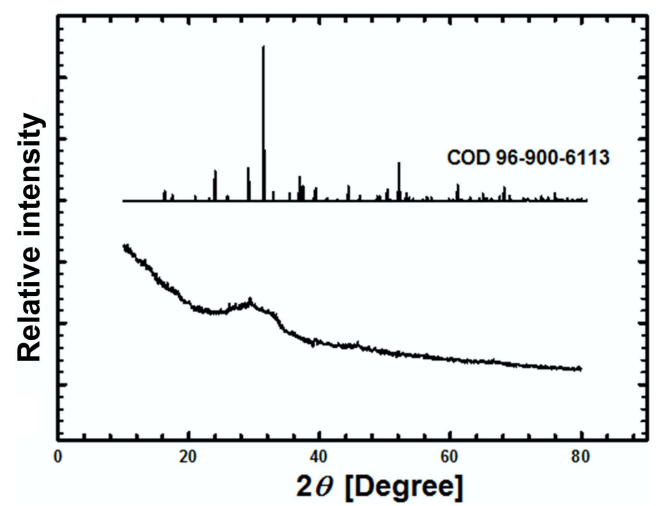

Fig. 2. X-ray diffraction patterns of gehlenite microspheres (down) and crystalline gehlenite sample from Crystallography Open Database.

XRD patterns confirm the amorphous nature of the microspheres.

The applied magnetic field $H_{a}$ dependences of the corrected mass magnetization $M^{\prime}$ at $300 \mathrm{~K}$ and $2 \mathrm{~K}$ are in Fig. 3. Both curves show practically no hysteresis. However, the magnetization at $300 \mathrm{~K}$ shows a diamagnetic, whereas the one at $2 \mathrm{~K}$ indicates a paramagnetic behaviour. It was found out that the paramagnetic or diamagnetic behaviour is a function of temperature and magnetic field.

The dependence of the magnetization $M^{\prime}$ vs. the applied magnetic field $H_{a}$ up to high magnetic field of $5.6 \mathrm{MA} \mathrm{m}^{1-}$ at $30 \mathrm{~K}$ is shown in Fig. 4. At $30 \mathrm{~K}$, the diamagnetic behaviour of the magnetization is evident at high magnetic field, whereas for the magnetic field near zero, a paramagnetic contribution and weak hysteresis can be observed; see the inset in Fig. 4 .

The temperature dependences of the corrected zerofield cooling (ZFC) and FC dc molar magnetic susceptibility $\chi$ are in Fig. 5 .

At the magnetic field of $4 \mathrm{kA} \mathrm{m}^{1-}$, the $\mathrm{ZFC}$ and $\mathrm{FC}$ curves show a significant bifurcation that suggests superparamagnetic or spin glass-like behaviours. The increase of the magnetic field to $0.8 \mathrm{MA} \mathrm{m}^{1-}$ results in the suppression of the bifurcation and decrease of the ZFC and

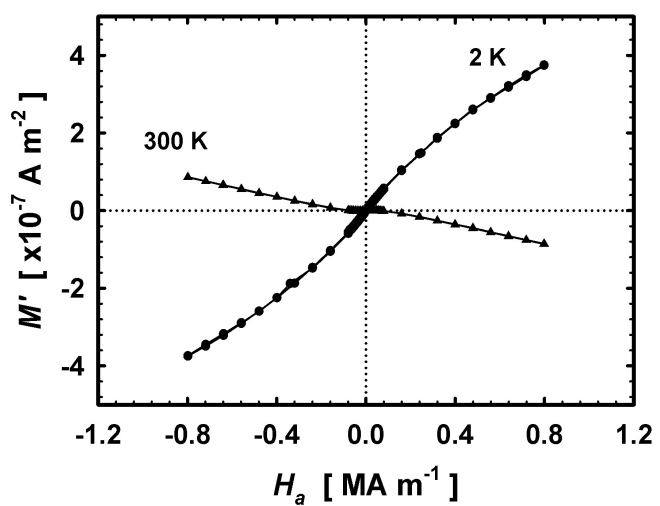

Fig. 3. $M^{\prime}$ vs. $H_{a}$ dependences of gehlenite microspheres at $300 \mathrm{~K}$ and $2 \mathrm{~K}$.

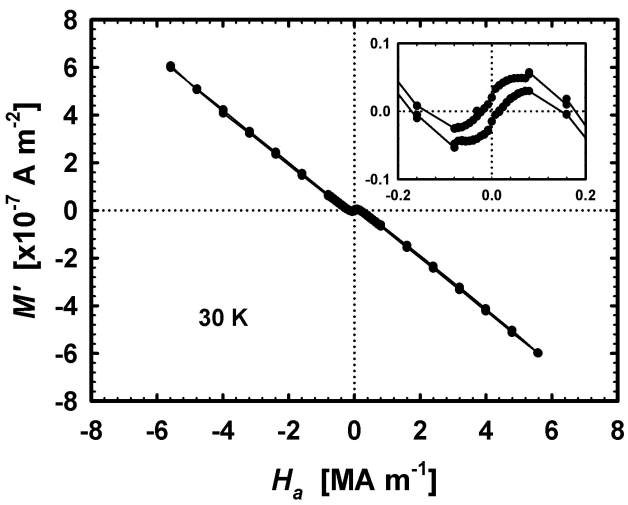

Fig. 4. $M^{\prime}$ vs. $H_{a}$ dependences of gehlenite microspheres at high applied magnetic field and at $30 \mathrm{~K}$. The inset shows an enlarged view at near zero magnetic field.

FC curves. Furthermore, the ZFC and FC curves show weak temperature dependence at high temperature suggesting the presence of temperature independent contribution $\chi_{T N}$.

Therefore, to determine the temperature independent contribution $\chi_{T N}$, we constructed $\chi T$ vs. $T$ dependences and determined the $\chi_{T N}$ from the slope of the curve of

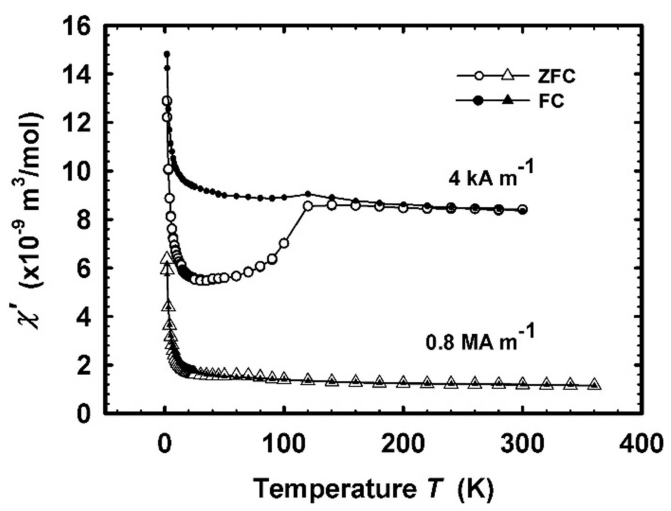

Fig. 5. Temperature dependences of the ZFC (empty) and the FC (full symbols) dc molar magnetic susceptibility $\chi$ of gehlenite miscrospheres at $4 \mathrm{kA} \mathrm{m}^{1-}$ (circles) and $0.8 \mathrm{MA} \mathrm{m}^{1-}$ (triangles). 
the linear fit of the dependences. The FC curves of $\chi(T)$ (see Fig. 5) after correction on $\chi_{T N}, \chi^{a}$, where $\left(\chi^{a}=\right.$ $\left.\chi-\chi_{T N}\right)$ are shown in Fig. 6.

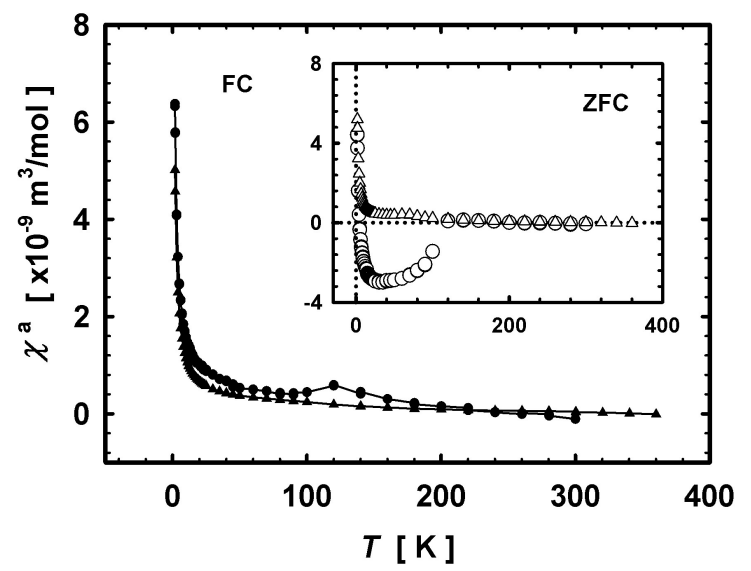

Fig. 6. Temperature dependences of FC molar dc magnetic susceptibility $\chi^{a}(T)$ (corrected for the temperature independent contribution $\chi_{T N}$ ) of gehlenite miscrospheres at $4 \mathrm{kA} \mathrm{m}^{1-}$ (full circles) and $0.8 \mathrm{MA} \mathrm{m}^{1-}$ (full triangles). The inset shows corresponding values of ZFC molar magnetic susceptibility $\chi^{a}(T)$ at $4 \mathrm{kA} \mathrm{m}^{1-}$ (open circles) and $0.8 \mathrm{MA} \mathrm{m}^{1-}$ (open triangles).

The curves FC $\chi^{a}(T)$ corrected to the temperature independent contributions $\left(\chi^{a}=\chi-\chi_{T N}\right)$ were fitted by the Curie-Weiss law $(\chi=C /(T-\Theta))$ separately; the first, for the temperature range of $120 \mathrm{~K}-220 \mathrm{~K}$ and the second for low temperature of $1.8 \mathrm{~K}-15 \mathrm{~K}$. In the high temperature range, the corresponding values of the Weiss temperature $\Theta^{a}$ are positive about $100 \mathrm{~K}$ and $65 \mathrm{~K}$ at magnetic field $4 \mathrm{kA} \mathrm{m}^{1-}$ and $0.8 \mathrm{MA} \mathrm{m}^{1-}$, respectively. In the low temperature range of $1.8 \mathrm{~K}-15 \mathrm{~K}$, the Weiss temperature values $\Theta^{b}$ are negative and near to zero $(\approx-1.9 \mathrm{~K}$ and $-1.0 \mathrm{~K}$, respectively).

The corresponding effective magnetic moments $\mu_{\mathrm{eff}}^{a}$ and $\mu_{\mathrm{eff}}^{b}$ are about $0.1 \mu_{\mathrm{B}}$ for both values of magnetic field. The change of sign of the Weiss temperature $\Theta$ with temperature signals a change in magnetic ordering from the ferromagnetic to the antiferromagnetic ordering with decrease of the temperature. An open question is what inner structure units of the amorphous gehlenite microspheres could be related or are relevant for the observed change of magnetic behaviours.

In this connection, we remind the results of Noller et al. [3] that reported a slight magnetization hysteresis at $298 \mathrm{~K}$ for the Fe-doped gehlenite, and the growth of the magnetic susceptibility with decrease of temperature up to $4 \mathrm{~K}$. The observed magnetic properties are attributed by them to magnetic domains, consisting of mutually interacting antiparallel spins and magnetic ordering (high value of the maximum magnetization) observed at low temperatures to their increasing mutual interactions. They left open the question of the role of magnetic domains in relation to the cationic tetrahedron structural units.
Magnetic properties of the amorphous gehlenite glass prepared in flame synthesis, especially the presence of $\chi_{T N}$, signal the existence of a non-bridging oxygen and oxygen vacancies (larger ring structures), taken into account local composition fluctuations, and perhaps even the trapped electrons or the electron anions [4-6].

\section{Conclusions}

The synthetic gehlenite $\left(\mathrm{Ca}_{2} \mathrm{Al}_{2} \mathrm{SiO}_{7}\right)$ amorphous microspheres were prepared by the $\mathrm{CH}_{4}-\mathrm{O}_{2}$ flame synthesis of precursor that was prepared from a stoichiometric mixture of high-purity powders $\mathrm{CaCO}_{3}, \mathrm{Al}_{2} \mathrm{O}_{3}$ and $\mathrm{SiO}_{2}$ annealing at $1000^{\circ} \mathrm{C}$ for $4 \mathrm{~h}$ by a standard solid-state reaction method. The magnetic properties of the microspheres were studied in detail by QD SQUID magnetometer MPMS XL-7. The gehlenite microspheres show a complex magnetic behaviour: the diamagnetism (at $300 \mathrm{~K}$ ) or paramagnetism (at $2 \mathrm{~K}$ ). This behaviour is a function of temperature and magnetic field. In addition, the significant effect of the applied magnetic field on the corrected ZFC and FC molar magnetic susceptibility was found. This can be ascribed to the presence of temperature independent contributions in the molar susceptibility. The independent susceptibility contributions indicate the presence of itinerant carriers. More features of spin glass were observed, such as the bifurcation of temperature dependences of ZFC and FC characteristics of molar magnetic susceptibility or low (near to zero) value of the effective magnetic moment obtained after correction of the molar magnetic susceptibility to the temperature independent contributions.

\section{Acknowledgments}

This work was supported by the projects VEGA 2/0152/13 and VEGA 2/0058/14 and by the Agency of the Ministry of Education of the Slovak Republic for the Structural Funds of the EU, Project Code 26240120019.

\section{References}

[1] M. Moesgaard, R. Keding, J. Skibsted, Y. Yue, Chem. Mater. 22, 4471 (2010).

[2] G.A. Bain, J.F. Berry, J. Chem. Educ. 85, 532 (2008).

[3] R. Nöller, H. Knoll, Solid State Commun. 47, 237 (1983).

[4] K.D. Vargheese, A. Tandia, J.C. Mauro, J. Chem. Phys. 132, 194501 (2010).

[5] A. Novatski, A. Steimacher, A.N. Medina, A.C. Bento, M.L. Baesso, L.H.C. Andrade, S.M. Lima, Y. Guyot, G. Boulon, J. Appl. Phys. 104, 094910 (2008).

[6] S.W. Kim, T. Shimoyama, H. Hosono, Science 333, 71 (2011). 